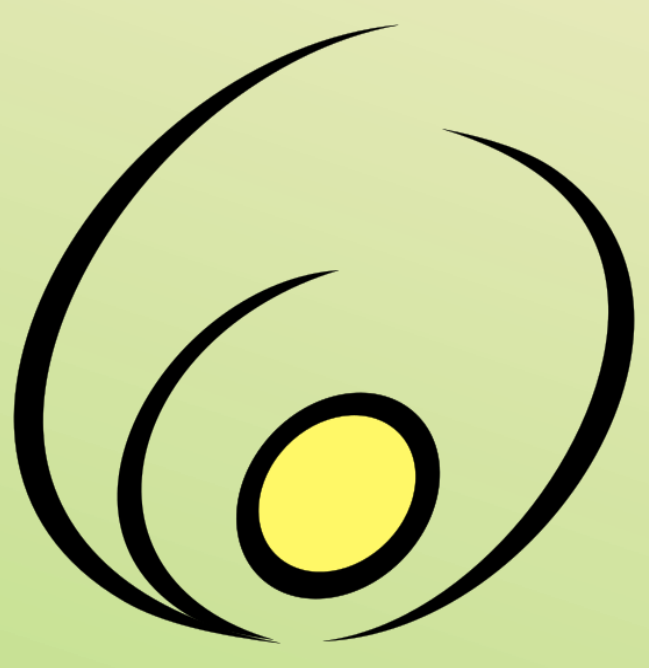

Fórum de

\section{Pró-Reitores}

\section{de Extensão}

\section{das Instituições}

Públicas de

Educação Superior

Brasileiras
Open access $\delta$ free available online

Revista Brasileira de Extensão Universitária

v. 8, n. 3, p. 159-165 set.- dez. 2017 e-ISSN 2358-0399

DOI: https://doi.org/10.24317/2358-0399.2017v8i3.5015

\title{
A Importância do Ensino de Anatomia Humana na Formação de Profissionais do Corpo de Bombeiros Militar
}

\author{
Karina Aparecida Resende ${ }^{1}$, Daniel Teixeira Machado², \\ Karine Joice Faria ${ }^{2}$, Lorena Rodrigues de Sena ${ }^{3}$, \\ Mariana Moreira Diniz ${ }^{2}$, Maira de Castro Lima ${ }^{4}$
}

Resumo: A anatomia humana é uma disciplina fundamental na formação de profissionais da saúde e socorristas. O conhecimento das estruturas corporais facilita a capacidade de compreensão, desenvolvimento e aplicações de técnicas de resgate, aprimorando assim o atendimento oferecido à população. Tendo em vista a importância dos socorristas no resgate às vítimas de diversos tipos de trauma, justifica-se a colaboração entre universidade e profissionais que trabalham nesta função para o ensino de anatomia humana. O projeto foi desenvolvido junto aos integrantes do $10^{\circ}$ Batalhão do Corpo de Bombeiros Militar de Minas Gerais que participaram de um projeto de extensão da Universidade Federal de São João del-Rei. Os acadêmicos prepararam o material didático (apostilas, aulas teóricas e roteiros de aula prática). Os sistemas estudados foram respectivamente, nervoso, circulatório, respiratório e osteomuscular. As aulas teóricas foram ministradas nas dependências do Corpo de Bombeiros e as práticas desenvolvidas no Laboratório de Anatomia Humana da UFSJ. Com o propósito de avaliar o conhecimento prévio e aprendizado dos bombeiros, antes e após as aulas teóricas, foi aplicado um teste, formulado pelos extensionistas, contendo dez questões fechadas cuja pontuação foi posteriormente analisada pelo Teste $t$ de Student pareado. Houve diferença estatística na porcentagem de acerto nas provas antes e após as aulas $(P<0,05)$. O projeto de extensão mostrou-se capaz de instruir os socorristas quanto à estrutura dos sistemas humanos estudados, auxiliando dessa forma no entendimento das técnicas de salvamento, o que pode refletir diretamente na melhoria dos serviços oferecidos à população.

Palavras-chave: Educação Continuada, Relações Comunidade-Instituição, Disciplinas das Ciências Biológicas

Content shared under Creative Commons Attribution 3.0 Licence CC-BY

1 Universidade Federal de São João del-Rei - Campus Centro-Oeste Dona Lindu - Curso de Farmácia, bolsista do programa Intitucional de Bolsas de Extensão PIBEX UFSJ.

2 Universidade Federal de São João del-Rei - Campus Centro-Oeste Dona Lindu - Curso de Medicina.

3 Universidade Federal de São João del-Rei - Campus Centro-Oeste Dona Lindu - Curso de Enfermagem.

4 Professora adjunta da Universidade Federal de São João del-Rei - Campus Centro-Oeste Dona Lindu. Coordenação Acadêmica mairacastrolima@ufsj.edu.br (autora para correspondência). 


\title{
The importance of human anatomy teaching in the training of fire department members
}

\begin{abstract}
Human anatomy is a fundamental discipline in the training of health professionals and rescuers. The knowledge of body structures facilitates the ability to understand, develop and apply rescue techniques, improving the care offered to the population. Taking into consideration the importance of rescuers on helping victims of various types of trauma, the collaboration between the university and the rescuer professionals for the teaching of human anatomy is justified. The project was developed with the members of the tenth battalion of the Minas Gerais Military Fire Brigade who participated in an Extension project of the Federal University of São João del-Rei. The academics prepared the didactic material (handouts, theoretical lessons, and practical lesson scripts). The studied systems were, respectively, nervous, circulatory, respiratory and musculoskeletal, the theoretical classes were given at the Fire Department and the practice was developed at UFSJ's Human Anatomy Laboratory. In order to evaluate the previous knowledge and the firefighter's learning, before and after the theoretical classes, a test formulated by students containing ten questions that were later analyzed through the paired Student $\mathrm{t}$-Test was applied to the participants. There was a statistical difference in the percentage of correctness in the tests before and after the classes were given $(P<0.05)$. The extension project was able to instruct rescuers on the structure of the organic systems studied, thus helping to understand the rescue techniques, which may reflect the improvement of the services offered to the population.
\end{abstract}

Keywords: Education, Continuing, Community-Institutional Relations, Biological Science Disciplines

\section{La importancia de la enseñanza de la anatomía humana en la formación de los} profesionales de cuerpo de bomberos

Resumen: La anatomía humana es una disciplina fundamental en la formación de los profesionales de la salud y de primera respuesta. El conocimiento de las estructuras del cuerpo facilita la capacidad de comprensión, desarrollo y aplicaciones técnicas de rescate y hace mejor el servicio ofrecido a la población. Dada la importancia de los equipos de rescate en responder en el socorro de las víctimas de diferentes tipos de trauma, se hizo necesario la colaboración entre la universidad y la corporación para la enseñanza de la anatomía humana a los miembros del Décimo Batallón de Minas Gerais, Brasil, cuerpo de bomberos participantes en un proyecto de extensión de la Universidad Federal de Sao João del-Rei (UFSJ). Los estudiantes académicos prepararon los materiales didácticos (libros de texto, clases y las apostillas de clases prácticas). Los sistemas estudiados fueron, respectivamente, nervioso, circulatorio, respiratorio y osteo-muscular, las clases teóricas fueron desarrolladas en las instalaciones del Departamento de Bomberos y las clases prácticas, desarrolladas en el Laboratorio de Anatomía Humana de la UFSJ. Con el fin de evaluar el conocimiento previo y el aprendizaje de los participantes, antes y después de las clases, se aplicó una prueba formulada por los estudiantes que contiene diez preguntas, que posteriormente fueron analizadas mediante la prueba $\mathrm{t}$ de Student para datos apareados. Hubo diferencia significativa estadísticamente en el porcentaje de respuestas correctas en las pruebas antes y después de las clases $(P<0,05)$. El proyecto de extensión ha demostrado ser capaz de educar a los equipos socorristas en responder sobre la estructura de los sistemas de órganos estudiados, ayudando así a la comprensión de las técnicas de rescate, lo que posiblemente refleja directamente la mejora de los servicios ofrecidos a población.

Palabras-clave: Educación Continua, Relaciones Comunidad-Institución, Disciplinas de las Ciencias Biológicas

\section{Introdução}

A Anatomia estuda, macroscopicamente, os componentes que constituem o seres organizados, "ana" significa partes e "tome", cortar, ou seja, cortar em partes. É uma ciência descritiva que compreende as formas do corpo humano utilizando nomes, conhecidos como termos anatômicos (DANGELO; FATTINI, 2007).

Por sua relevância é considerada uma disciplina básica na formação de profissionais da saúde e socorristas. Esse conteúdo permite a compreensão das estruturas que compõem o corpo, auxiliando no entendimento das patologias, nos processos de tratamento e intervenção (MEC/SESU, 2010).

O socorro de urgência, como o realizado pelos bombeiros, são medidas primordiais realizadas fora do ambiente hospitalar, com objetivo de reduzir danos e agravos à saúde de uma vítima que sofreu algum tipo acidente ou um mal súbito (RIBEIRO, 2011, LEMOS et al., 2012).

$\mathrm{O}$ atendimento dos socorristas do corpo de bombeiros se resume ao suporte básico de vida, com uso de oxigênio medicinal e desfibrilador externo semiautomático como recursos de intervenção direta. Durante o processo de 
socorro é dada prioridade à manutenção das vias aéreas pérvias, controle de hemorragias, estabilização dos pacientes, e imobilizações que se fizerem necessárias (MINAS GERAIS, 2013).

O serviço de socorro deve ser adaptado para diferentes vítimas de acordo com a composição corporal e o conhecimento da anatomia permite que essa adaptação seja realizada de maneira adequada. Um estudo que estimou a relação ótima de compressões torácicas para ventilação na ressuscitação cardiopulmonar (RCP), a fim de maximizar o aporte sistêmico de oxigênio, concluiu que a relação compressão/ventilação na RCP deve ser menor para as crianças do que para os adultos, e aumentado gradualmente como uma função do peso corporal (BABBS; NADKARNI, 2004).

O conhecimento das estruturas anatômicas também permite que o decúbito do paciente seja determinado de maneira correta e segura durante atendimento. Em certas situações de urgência, como alguns tipos de traumas, o indivíduo precisa manter-se em posição estabelecida, dessa forma é impossibilitado de realizar mudanças de decúbito sem técnica, e inúmeras estratégias precisam ser realizadas para transporte em diferentes posições. A manipulação das vítimas de forma incorreta pode acarretar inúmeros danos para o indivíduo (FIORUC, 2010).

Um atendimento preciso, no qual os socorristas dominam as técnicas e estão bem capacitados para prestar atividades de socorro, pode contribuir de forma positiva na diminuição das taxas de mortalidade e redução das chances de sequelas graves, resultantes de um atendimento não apropriado (PEREIRA; LIMA, 2009, RIBEIRO, 2011). Levando em consideração a importância do corpo de bombeiros no socorro a vítimas de diversos tipos de acidentes, o entendimento das estruturas anatômicas aprimora o atendimento oferecido à população. A educação em anatomia é primordial para o profissional que deseja manter-se atualizado e oferecer um trabalho de qualidade à sociedade (CARDINOT et al., 2014).

Ao oferecer aos socorristas a oportunidade de ampliar e aprofundar seus conhecimentos sobre anatomia humana, o projeto de extensão teve como objetivo promover o aumento do entendimento das estruturas corpóreas pelos socorristas integrantes do projeto. Todo conhecimento teórico repassado para os bombeiros facilita no momento do socorro fazerem adaptações de suas técnicas, melhorando a qualidade e eficiência dos serviços prestados por eles.

\section{Métodos}

As atividades de extensão foram desenvolvidas no período de abril de 2015 a março de 2016, por cinco acadêmicos do curso de enfermagem, farmácia e medicina da Universidade Federal de São João del-Rei Campus Centro-Oeste Dona Lindu (UFSJ/CCO), em Divinópolis - MG, sob orientação e supervisão da docente responsável. Os estudantes envolvidos no projeto foram capacitados para ensinar anatomia em aulas teóricas e práticas. Foram realizadas reuniões semanais para o estudo de livros, atlas e artigos científicos da área de anatomia humana e posterior preparação de material didático (apostilas, aulas teóricas e roteiros de aula prática).

Os alunos extensionistas prepararam as aulas teóricas e práticas por meio de estudos semanais. O teste de múltipla escolha, aplicado antes e após as aulas teóricas, foi formulado pelos universitários e contemplou o conteúdo abordado na aula. Não houve avaliação com relação às aulas práticas. Os estudantes também formularam o questionário aberto aplicado após a última aula prática com o objetivo de avaliar a percepção dos bombeiros com relação ao projeto.

O público alvo do projeto foram os bombeiros integrantes do $10^{\circ}$ Batalhão de Bombeiros Militar de Minas Gerais, que se interessaram em adquirir conhecimento sobre anatomia humana. As aulas teóricas aconteceram nas dependências do corpo de bombeiros e as aulas práticas no Laboratório de Anatomia Humana da $\mathrm{UFSJ} / \mathrm{CCO}$.

Os sistemas humanos, abordados durante o curso, seguiram a ordem: o primeiro módulo, sistema nervoso, composto por 5 horas de aula teórica e 3 horas de aula prática. $\mathrm{O}$ segundo, sistema circulatório, por 4 horas de aula teórica e 3 horas de prática. O terceiro, sistema respiratório, por 2 horas de aula teórica e 2 horas de prática. $\mathrm{O}$ último, sistema osteomuscular, por 3 horas de aula teórica e 4 horas de aula prática. Esses sistemas humanos foram estudados por serem os mais relacionados com o atendimento de urgência e emergência em casos de ameaça a vida.

Com o objetivo de mensurar o grau de conhecimento prévio dos bombeiros sobre os sistemas humanos e avaliar o impacto da qualidade do ensino oferecido pelo projeto, foi aplicado um teste, antes e após as aulas teóricas, contendo dez questões de múltipla escolha referentes ao sistema estudado. Esse teste foi elaborado pelos universitários extensionistas. A proporção de acertos foi analisada pelo teste $t$ de Student pareado. Para comparação das notas nas provas teóricas após as aulas, entre os diferentes sistemas biológicos do corpo humano, foi utilizada a ANOVA de um fator (One Way Anova), seguida de o teste de comparações múltiplas de Tukey (Tukey's post hoc test).

Ao fim do projeto, foi elaborado um questionário com cinco perguntas abertas, em que foram solicitadas aos participantes observações, críticas positivas e negativas, com relação ao projeto de extensão. Essas perguntas foram respondidas pelos socorristas após a última aula prática. As respostas das questões abertas foram consideradas para promover melhoras nas aulas e orientar a condução do projeto futuramente. Pode-se perceber o grau de satisfação dos bombeiros com relação ao conteúdo das aulas, aplicabilidade do conteúdo à prática de socorro e necessidade de adaptações no projeto. 


\section{Resultados}

Cada aula, teórica e prática, teve a participação de cerca de 20 bombeiros. Todos os integrantes da corporação, cerca de 160, foram convidados. Somente aqueles interessados em educação continuada em anatomia e com o horário disponível participaram das aulas.

Foi elaborado pelos alunos, durante o desenvolvimento do projeto, um material de didático (slides) e uma apostila contendo tópicos de todos os sistemas estudados.

Os resultados dos testes, nas aulas teóricas, foram apresentados na forma de porcentagem, na qual se obteve a média e erro padrão da média dos acertos antes e após os estudos. Para todos os sistemas estudados, a comparação de acertos antes e após as aulas teóricas apresentou diferença estatisticamente significativa. O teste t de Student pareado foi utilizado para avaliar o número de acertos de cada sistema orgânico. Com relação ao sistema nervoso a porcentagem de acertos antes das aulas foi de 32,7\% e depois 58,2\%, $(P=0,017)$, sistema circulatório: antes $77 \%$ e depois $91 \%,(P$ $=0,024)$, sistema respiratório: antes $51 \%$ e depois $-86 \%$, $(P=0,012)$ sistema osteomuscular: antes $67 \%$ e depois 91\%, $(P=0,0007)$, Figura 1.

Uma vez terminado o módulo, os bombeiros que atingiram $60 \%$ de aproveitamento no teste após a aula teórica receberam um certificado referente ao período de estudo. Aqueles que participaram dos quatro módulos e foram aprovados em todos receberam um certificado de conclusão do curso. Os reprovados foram convidados a continuar no projeto e aprofundar os estudos no sistema humano em que não obtiveram resultado satisfatório
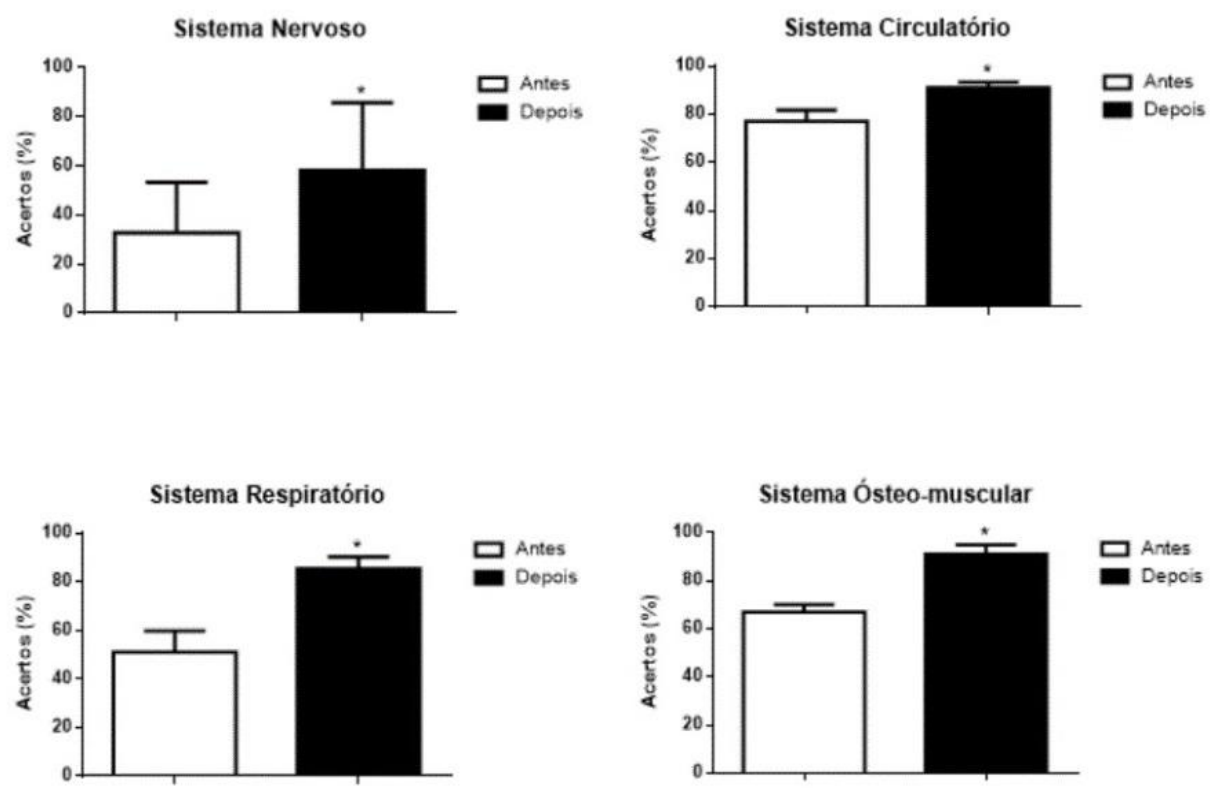

Figura 1: Porcentagem de acerto nos testes sobre os sistemas estudados, antes e após as aulas teóricas; todos os sistemas apresentaram maior proporção de acertos depois, segundo o teste $t$ de Student para dados pareados $(P<0,05)$.

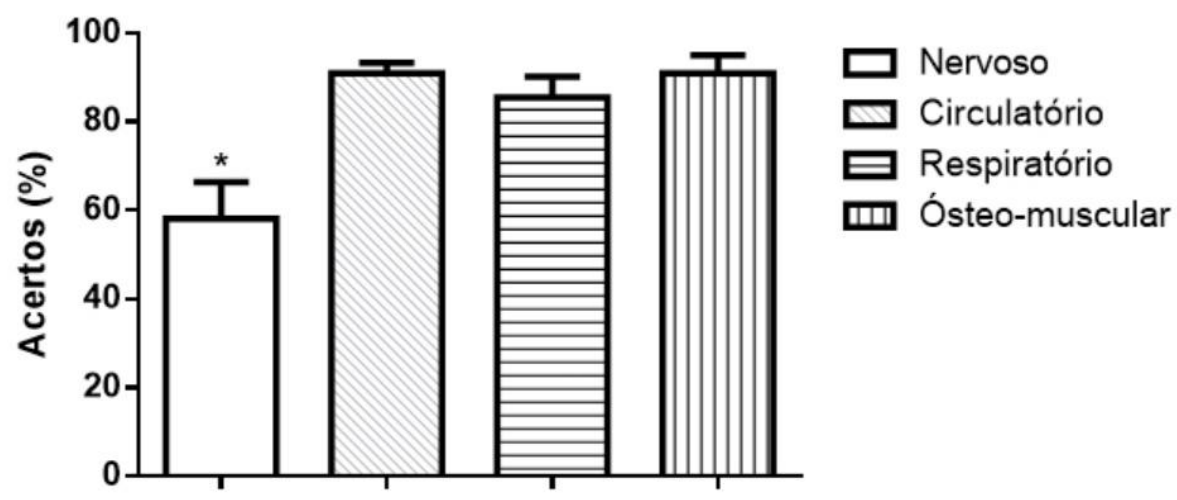

Figura 2: Porcentagem de acertos nos testes, após as aulas teóricas de anatomia, comparada entre os diversos sistemas orgânicos estudados (ANOVA de um fator, $P=0,0002$ ). $O$ asterisco indica diferença entre o sistema nervoso e os demais, segundo o teste de Tukey $(\mathrm{P}<0,05)$ 


\section{Discussão}

Os extensionistas tiveram oportunidade de aprendizado com o projeto, o que corrobora o resultado de estudos anteriores. LEMOS et al. (2012), relata a experiência de alunos do curso de medicina no ensino e treinamento sobre princípios básicos de primeiros socorros em escolas. Conclui-se que o projeto foi capaz de impactar de forma positiva a formação do estudante quando ele pratica a educação em saúde.

O acadêmico foi colocado como sendo o protagonista no desenvolvimento do trabalho. Nesse aspecto, o estudante deixou de ser apenas receptor e passou a ser participante do processo. Permitindo reconhecer-se como responsável em praticar ações que visam cumprir deveres e direitos que promovam transformação social de acordo com a Política Nacional de Extensão Universitária (2012).

$\mathrm{Na}$ análise dos valores de porcentagem dos testes de múltipla escolha, antes e após as aulas teóricas, observou-se que os bombeiros que participaram do projeto têm grande conhecimento acerca das estruturas relacionadas ao sistema circulatório, o que pode ser explicado pelo fato da maioria das ocorrências estarem ligadas a esse sistema. Alguns bombeiros possuem formação de nível superior, o que pode ter contribuído para o alto índice de acerto em alguns sistemas humanos.

Constatou-se que os bombeiros possuíam pouco conhecimento prévio sobre o sistema nervoso e mesmo após as aulas, o total de acertos ainda não alcançou $60 \%$. A estrutura do sistema nervoso é complexa e vários atendimentos são relacionados ao mesmo. O próprio tenente da corporação solicitou prioridade no estudo dessas estruturas, tendo em vista a sua importância em situações de socorro. Os resultados mostraram esse déficit real na formação dos soldados.

A educação continuada deve ser incentivada para estudantes e profissionais socorristas formados com o intuito de servir à sociedade. CALLIS et al. (2016), mostraram que alunos de medicina que participaram de um curso extracurricular de primeiros socorros estavam aptos a atuar como socorristas voluntários em caso de desastre.

Inúmeras variáveis podem comprometer a qualidade do atendimento em primeiros socorros. Por exemplo, a fadiga do socorrista pode interferir na eficácia da manobra de ressuscitação cardiopulmonar - RCP (GIANOTTO-OLIVEIRA et al., 2015). Informações resultantes de investigação científica podem modificar a dinâmica da equipe de socorro e potencializar resultados positivos. A atualização permanente do conhecimento do profissional pode garantir qualidade e eficácia no atendimento. Incentivar a educação continuada é um importante papel da universidade que deve interagir com a sociedade e promover uma constante troca de conhecimento.

O contato direto do aluno com o profissional socorrista produziu uma rica troca de informações e experiências, uma vez que os alunos possuem prévio conhecimento em relação à anatomia enquanto os bombeiros mostram-se capacitados no campo prático da profissão, essa união permitiu um aumento mútuo do conhecimento e integração entre ambas às partes. Docente e acadêmicos aprenderam com as questões práticas discutidas durante as aulas sobre primeiros socorros e técnicas de salvamento. Abaixo, algumas respostas dos participantes do projeto, ao questionário aplicado na última aula prática.

\section{[...] “A continuidade do projeto contribuirá ainda mais na ampliação de nossos conhecimentos, ajudando na prestação de socorro (assistência), com mais eficiência $e$ tranquilidade."}

[...] “Com os módulos ministrados pude aprender um pouco mais e acho que com isso todos ganharam principalmente a sociedade"

O material elaborado pelos alunos foi encaminhado ao $10^{\circ}$ Batalhão de bombeiros e servirá como referencial teórico nas aulas do curso de formação dos bombeiros militares que posteriormente pertencerão a este batalhão bem como os bombeiros que não conseguiram participar das aulas.

$\mathrm{Na}$ literatura, outros estudos também descrevem a produção de material didático. No trabalho de DA SILVA et al. (2016), acadêmicos de cursos superiores abordaram conhecimentos sobre ciências biológicas em escolas públicas, e foi relatado que por meio dos resultados positivos alcançados durante o projeto de extensão foi solicitado que os coordenadores enviassem material didático complementar para que os demais alunos da escola que não participaram, por diversas razões, pudessem ter acesso ao conteúdo. Sendo assim, é possível supor que as experiências vivenciadas no presente trabalho poderão contribuir para a formação de outras pessoas através do ensinamento pelos próprios bombeiros.

Pelo seu importante papel o projeto foi amplamente divulgado pela imprensa local sendo citado por seis sites de notícias. Foi realizada também uma entrevista para o Telejornal TV Alterosa do Centro-Oeste de Minas, que teve como objetivo divulgar o projeto para a comunidade na perspectiva de mostrar que a universidade está disponível e aberta à troca de saberes (G37 s/d, GLOBO, s/d, PORTAL CENTRO-OESTE s/d, PORTAL MPA s/d, UFSJ s/d, YOUTUBE s/d). O projeto recebeu menção honrosa no XIV Congresso de produção científica e acadêmica da UFSJ. Foi solicitada intenção de expandir o projeto para polícia militar de Minas Gerias e equipes de socorristas do município.

\section{Conclusão}

O projeto de extensão de ensino da anatomia para os militares do corpo de bombeiros foi implantado com êxito. Os participantes tiveram aumento do conhecimento referente às estruturas do corpo humano e os universitários extensionistas foram capacitados no aprendizado e ensino de anatomia. 
O conhecimento das estruturas orgânicas transmitido ao profissional socorrista pode aumentar a probabilidade de sucesso em uma manobra de salvamento. As atividades desenvolvidas mostraram que a comunidade universitária pode efetivamente intervir e promover mudanças na sociedade. Esse projeto pode ser repetido em diferentes partes do país, uma vez que a anatomia faz parte do ciclo básico da formação de cursos da área da saúde.

\section{Agradecimentos}

À Universidade Federal de São João Del-Rei, ao $10^{\circ}$ Batalhão do Corpo de Bombeiros Militar de Minas Gerais - Divinópolis-MG, e e ao apoio da Pró-Reitoria de Extensão e Assuntos Comunitários - PROEX.

\section{Contribuição de Cada Autor}

K. A. R. gerenciou as atividades realizadas no desenvolvimento do trabalho. Preparou e ministrou as aulas de anatomia para os militares. Escreveu o texto final. D. T. M. preparou e ministrou as aulas de anatomia para os militares. Escreveu o texto final. K. J. F. preparou e ministrou as aulas de anatomia para os militares. Formatou todas as aulas preparadas em PowerPoint. Contribuiu para escrita e revisou o texto final. L. R. S. e M. M. D. prepararam e ministraram as aulas de anatomia para os militares. Colaboraram com a análise estatística e revisão do texto final. M. C. L. atuou como coordenadora e orientadora dos bolsistas e voluntários, além da orientação de escrita do artigo científico.

\section{Referências}

BABBS, C. F.; NADKARNI V. Optimizing chest compression to rescue ventilation ratios during onerescuer CPR by professionals and lay persons: children are not just little adults. Resuscitation, v. 61, n. 2, p. 173-181, 2004.

CALLIS, G. L. P.; CASTELLANO, R. D. D.; MACÍAS, S. P.; DEL TORO BASULTO, C. Curso extracurricular de primeros auxilios a estudiantes de medicina para el aprendizaje de socorrismo. Medisan, v. 20, n. 6, p. $761,2016$.

CARDINOT, T. M.; OLIVEIRA, J. D.; JÚNIOR, O. V. P.; MACHADO, M. A.; MACEDO, M. D.; ARAGÃO, A. D. Importância da Disciplina de Anatomia Humana para os Discentes de Educação Física e Fisioterapia da ABEU Centro Universitário de Belford Roxo- RJ. Coleção Pesquisa em Educação Física, v. 13, n.1, p. 95-101, 2014.

DA SILVA, A. M.; ZANESCO, C.; CAZAROTTO, A. R.; BORSOI, F. T.; DERVANOSKI, C.; MAROLLI, C.; ZANELLA, K. A.; SILVA, D. T. R.; BAGATINI, M. D. $\mathrm{O}$ ensino de Ciências Biológicas uma experiência teórico prática com alunos do ensino médio de escolas públicas.
Revista Brasileira de Extensão Universitária, v. 7, n. 2, p. 99-104, 2016.

DANGElO, J.G., FATTINI, C.A. Anatomia Humana: sistêmica e segmentar. 3. ed. São Paulo: Atheneu, 2007.

FIORUC, B. E.; MOLINA, A. C.; JUNIOR, W. V.; LIMA, S. A. M. Educação em saúde: abordando primeiros socorros em escolas públicas no interior de São Paulo. Revista Eletrônica de Enfermagem, v. 10, n.3, p. 695-702, 2008.

GIANOTTO-OLIVEIRA, R. GIANOTTO-OLIVEIRA, G.; GONZALEZ, M. M.; QUILICI, A. P.; ANDRADE, F. P., VIANNA, C. B.; TIMERMAN, S. Quality of continuous chest compressions performed for one or two minutes. Clinical Science, v. 70, n. 3, p. 190-195, 2015.

G37. Projeto da UFSJ ensina anatomia a integrantes do corpo de bombeiros em Divinópolis. G37, Divinópolis, out. de 2015. Disponível em:< https://g37.com.br/c/ divinopolis/projeto-da-ufsj-ensina-anatomia-aintegrantes-do-corpo-de-bombeiros-em-divinopolis > Acesso em: 16 mar. 2016.

GLOBO. Projeto da UFSJ ensina anatomia a grupo dos bombeiros em Divinópolis. Disponível em: < http://g1.globo.com/mg/centro-oeste/noticia/2015/10/ projeto-da-ufsj-ensina-anatomia-grupo-dos-bombeirosem-divinopolis.html > Acesso em: 16 mar. 2016.

LEMOS, E. F. L.; NISIYAMA, A. L.; FARIAS, I. E. C. D.; HAMANN, E. M. Educação em Saúde: A experiência de alunos de medicina no ensino em primeiros socorros. Revista ParticipAção, n. 20, p. 3542, 2012.

MEC/SESU. Referências curriculares nacionais dos cursos de bacharelado e licenciatura. Brasília: Ministério da Educação: Secretária de Educação Superior, 2010.

MINAS GERAIS. Corpo de Bombeiros Militar de Minas Gerais. Instrução Técnica Operacional n. 23: protocolo de atendimento pré-hospitalar. Belo Horizonte: CBMMG, 2013. Disponível em: < http://www. bombeiros.mg.gov.br/component/content/article/20660instrucoes-tecnicas-operacionais.html > Acesso em: 17 mar. 2017.

PEREIRA, W. A. P.; LIMA, M. A. D. S. O trabalho em equipe no atendimento pré-hospitalar à vítima de acidente de trânsito. Revista da Escola de Enfermagem da USP, v. 43, n. 2, p. 320-7, 2009.

POLÍTICA NACIONAL DE EXTENSÃO UNIVERSITÁRIA. Fórum de Pró-Reitores de Extensão das Instituições Públicas de Educação Superior Brasileiras. Porto Alegre: Gráfica da UFRGS, 2012.

PORTAL CENTRO-OESTE. Projeto ensina anatomia a militares do corpo de bombeiros. Disponível em: < http://portalcentrooeste.com/projeto-ensina-anatomia-amilitares-do-corpo-de-bombeiros/ > . Acesso em: 18 mar. 2017.

PORTAL MPA. Curso da UFSJ ensina anatomia a militares do corpo de bombeiros em Divinópolis. Disponível em:< http://www.sistemampa.com.br/ 
\%20noticias/saude/curso-da-ufsj-ensina-anatomia-amilitares-do-corpo-de-bombeiros-em-divinopolis/ Acesso em: 18 mar. 2017.

RIBEIRO, C. S. Os primeiros socorros como uma competência de efetivação dos direitos referentes à vida e à saúde: o desafio do educador infantil. In: COLÓQUIO INTERNACIONAL DE EDUCAÇÃO, 3, e SEMINÁRIO DE PESQUISA SOBRE INDICADORES DE QUALIDADE DO ENSINO FUNDAMENTAL, 1. Anais... Joaçaba-SC, v.1, n.1 2011.

UFSJ. Anatomia para bombeiros em Divinópolis. Disponível em: < http://www.ufsj.edu.br/noticias ler.php?codigo noticia $=5658>$. Acesso em: $20 \mathrm{fev}$. 2017.

YOUTUBE. Bombeiros participam de projeto desenvolvido pela UFSJ. Disponível em: < https://www.youtube.com/watch?v=KCYcE1tQ4z8 >. Acesso em: 18 mar. 2017.

\footnotetext{
Como citar este artigo:

RESENDE, K. A.; MACHADO, D. T.; FARIA, K. J.; DE SENA, L. R.; DINIZ, M. M.; LIMA, M. de C. A Importância do ensino de anatomia humana na formação de profissionais do corpo de bombeiros militar. Revista Brasileira de Extensão Universitária, v. 8, n. 3, p. 159-165, 2017. Disponível em: < https://periodicos.uffs.edu.br /index.php/RBEU/article/view/5015/pdf >
} 